\title{
Do we at all need surgery to treat thrombosed external hemorrhoids? Results of a prospective cohort study
}

\author{
Ole Gebbensleben' \\ York Hilger ${ }^{2}$ \\ Henning Rohde ${ }^{3}$ \\ 'Park-Klinik Berlin-Weissensee, \\ Berlin, Germany; ${ }^{2}$ Institut für \\ Biostatistik, Freiburg im Breisgau, \\ Germany; ${ }^{3}$ Praxis für Endoskopie \\ und Proktologie, Berlin, Germany
}

Correspondence: Henning Rohde Praxis für Endoskopie und Proktologie, Viktoria-Luise-Platz I2, 10777 Berlin, Germany

Tel +4930364402 26

Fax +493021969268

Email mail@prof-rohde.de
This article was published in the following Dove Press journal:

Clinical and Experimental Gastroenterology

26 June 2009

Number of times this article has been viewed

Background: It is unknown whether surgery is the gold standard for therapy of thrombosed external hemorrhoids (TEH).

Methods: A prospective cohort study of 72 adults with TEH was conducted: no surgery, no sitz baths but gentle dry cleaning with smooth toilet paper after defecation. Follow-up information was collected six months after admission by questionnaire.

Results: Despite our strict conservative management policy 62.5\% (45/72) of patients (95\% confidence interval [CI]: 51.0-74.0) described themselves as "healed" or "ameliorated", and $61.1 \%(44 / 72,95 \% \mathrm{CI}: 49.6-72.6)$ found our management policy as "valuable to test" or "impracticable". $13.9 \%$ (10/72, 95\% CI: 5.7-22.1) of patients suspected to have recurrences. $4.2 \%$ did not know. Twenty-two of the 48 responding patients reported symptoms such as itching $(18.8 \%)$, soiling $(12.5 \%)$, pricking $(10.4 \%)$, or a sore bottom $(8.3 \%)$ once a month $(59.1 \%, 13 / 22)$, once a week $(27.3 \%, 6 / 22)$, or every day $(13.6 \%, 3 / 22)$.

Conclusions: The dictum that surgery is the gold standard for therapy for TEH should be checked by randomized controlled trials.

Keywords: hemorrhoids, acute hemorrhoidal disease, thrombosed external hemorrhoid, perianal thrombosis, conservative therapy, surgery

\section{Introduction}

Symptoms of benign anal diseases like internal hemorrhoids rank among the most common complaints of patients seen in primary care practices. ${ }^{1-4}$ Etiology of thrombosed external hemorrhoid (TEH) is unknown. ${ }^{5-8}$ Synonyms for thrombosed external hemorrhoid are acute thrombosed external hemorrhoid, ${ }^{8,9}$ acute hemorrhoidal disease, ${ }^{10}$ anal hematoma, ${ }^{11,12}$ perianal hematoma, ${ }^{13,14}$ thrombosed haemorrhoid, ${ }^{15}$ hemorrhoidal thrombosis, ${ }^{7,12}$ or perianal thrombosis. ${ }^{16,17}$ It was suggested to rename the disease "perianal thrombosis" to make it distinguishable from hemorrhoids since a causal connection is unproven. ${ }^{17}$ Histologically, thrombi are found in perianal veins, not in subcutaneous tissue and $d^{7,14,15,17}$ the term "hematoma" is wrong. . $^{212-14,17}$

TEH has two main modalities of clinical presentation: as a common single external pile or as a circular thrombosis of external hemorrhoids. This paper is concerned with a single TEH only following Hancock's definition of "an acute localized thrombosis which may affect the external plexus".2

TEH occurs accidentally and understandably patients take fright. ${ }^{2,3,17,19}$ As therapy, physicians inject local anesthesia into anal skin which is very painful, do 
an incision or excision, and then take thrombi out. ${ }^{2,7,15-17,20,21}$ Is this necessary? Only a minority of such patients present with formidable swelling, fierce bleeding, and overwhelming pain. Because patients fear surgery, they wait and observe their symptoms. Sometimes they present hours, even days after onset with less swelling, less pain, and no bleeding. Because swelling vanishes, a thrombus must not perforate anal skin, which means no bleeding, and may disappear within two to three weeks by resorption.

There are no randomized controlled trials comparing surgical and conservative management of THE, ${ }^{7,15,16,20}$ but surgical management is the gold standard ${ }^{10,20,22}$ if the condition is encountered within the first 72 hours after onset $^{3,23}$ or fails to respond to conservative treatment. ${ }^{2,8,18}$ What happens when a strict conservative management is started with painkillers, a wait-and-see policy, and dry anal cleaning after motions ${ }^{24,25}$ independent of stage of TEH at presentation?

\section{Methods}

Patients with TEH of both sex, aged 16-80 years, presenting at our office from March 18th, 2004 to August 18th, 2005 referred from general practitioners (GPs), physicians, urologists, or gynecologists because of anal complaints such pain or bleeding, were consecutively enrolled. After proctologic assessment in knee-chest position ${ }^{26}$ we informed patients that they had a benign lesion which would not need surgery. It would heal if patients were willing to accept our strict management policy: no water, shower, bath, washcloth, wet wipes, soap, or shower gel, but smooth dry toilet paper for anal cleaning after defecation for one to two weeks, and body cleaning without shower or bath tube but as often as wished with water, soap, or shower gel with an accepted washcloth for their inflamed anal skin to protect it against unknown etiologic factors which might postpone healing. ${ }^{24,25}$

\section{Questionnaires}

Individuals were asked to complete questionnaires with given answers about anal history and symptoms at study entry (Table 1) and six months later at follow-up (Table 2).

\section{Follow-up}

Patients were instructed to return to our practice immediately in case of problems. We phoned them six months later to deliver our follow-up questionnaire since we would like to learn how they are and the course of healing of their TEH.
Table I Patients' questionnaire with given answers at study entry

I. Which symptom or sign did you experience entering our outpatient clinic?

Anal lump

Anal pain

Anal burning (baking)

Anal itching

Anal bleeding

Anal pricking

Anal soreness

2. Which symptom bothered you most?

Anal lump

Anal pain

Anal burning (baking)

Anal itching

Anal bleeding

Anal pricking

Anal soreness

3. How long have you suffered from these symptoms or signs?

A few days

Up to one week

Two to four weeks

Up to half a year

Up to one year or longer

4. Did you experience a painful lump earlier?

No

Yes

Once

Repeatedly

Unknown

5. Did you treat your lesion by yourself?

No

Yes

6. Did you assume that you had hemorrhoids?

No

Yes

Unknown

7. Were you under medical treatment for hemorrhoids when your painful lump appeared?

No

Yes

8. How do you clean your anus after defecation?

Dry toilet paper

Water

Wet wipes

Shower

With soap or shower gel

9. How often do you shower?

Not at all

At least every day

Once or twice a week

More than twice a week

Once a month

(Continued) 
Table I (Continued)

10. How often do you take a bath tube?

Not at all

At least every day

Once or twice a week

More than twice a week

Once a month

\section{Statistics}

We completed intention-to-treat analyses. To compare anal cleaning attitudes at start and six months later, McNemar's test for related samples was used for significant difference for dichotomous variables and Wilcoxon signed-rank test for ordinal variables. P-values were computed using the exact versions of both tests. The Student's $t$-test for independent variables was used to find out which individuals had fewer symptoms: those who followed our strict management policy "more than one week" or "one week or less". We used SPSS software (v. 15.0.1.1; SPSS inc., Chicago, IL, USA).

\section{Ethical guidelines}

This study has been conducted in accordance with the Declaration of Helsinki (1964) and was conducted with the understanding and the consent of the patients.

\section{Results}

All 72 patients initially accepted our therapeutic regimen. Patient characteristics are summarized in Table 3.

Two patients called on us in the first two weeks after admission because of healing problems: a 32-year-old man was dissatisfied with prolonged healing, but we persuaded him into continuing therapy. A 73-year-old lady was seen repeatedly because of recurrent anal bleeding because of TEH. She was happy with our treatment policy because she escaped surgery (Figures 1A-F).

Median prevalence of TEH per month at our institution was 9 (5-14). A seasonal occurrence was found in springtime. Symptoms at admission were: anal lump (80.3\%), pain $(73.2 \%)$, burning (baking) (43.7\%), itching (42.3\%), bleeding (28.6\%), pricking (26.8\%), and/or anal soreness (16.9\%). Patients were bothered most by pain (43.5\%), lump (40.6\%), and anal blood (8.7\%). Onset of symptoms was within "some days" in $40.0 \%$ of patients, "one week" (34.3\%), "four weeks" (12.9\%), "half a year" (2.8\%), and "one year or longer" (10.0\%). Half of patients $(50.7 \%)$ had not experienced a painful lump earlier, some once (27.5\%), others repeatedly (21.7\%). 54.9\% of patients thought they might have hemorrhoids, $31.0 \%$ did not know, and
Table 2 Patients' questionnaire with given answers at follow up

I. Which symptom or sign do you experience?

Anal lump

Anal pain

Anal burning (baking)

Anal itching

Anal bleeding

Anal pricking

Anal soreness

2. How often have you suffered from these symptoms within the last days? I have no symptoms

Every day

Once a week

Once a month

3. Do you feel

Healed

Ameliorated

Or unchanged?

4. Do you think your anal lesion has healed?

No

Yes

Unknown

5. How long did you comply with our strict conservative management policy?

One week

More than one week

Not at all

Unknown

6. Did you experience a recurrence at the anus?

No

Yes, I suspected one

Unknown

7. What kind of therapy followed your suspected recurrence?

There was no recurrence

Surgery

Ointments and/or suppositories

I adopted your strict anal cleaning policy

8. What do you think about our strict conservative management policy? Valuable to test

Intelligible

Incomprehensible

Impracticable

Unknown

9. How do you clean your anus after defecation?

Dry toilet paper

Water

Wet wipes

Shower

With soap or shower gel

(Continued) 
Table 2 (Continued)

10. How do you clean your body?
Shower
Bath tube
Wash cloth
11. How often do you shower?
Not at all
At least every day
Once or twice a week
More than twice a week
Once a month
12. How often do you take a bath tube?
Not at all
At least every day
Once or twice a week
More than twice a week
Once a month

$14.1 \%$ declined. $56.4 \%$ of patients with assumed hemorrhoids tried to treat themselves. $29.4 \%$ had been under medical treatment for so called hemorrhoids when TEH appeared.

At the six-month follow-up (median, range 2-13 months) after admission only 48 out of 72 patients (66.7\%) sent their questionnaire back. $62.5 \%$ (45/72, 95\% confidence interval [CI]: 51.0-74.0) of patients described themselves as "healed" or "ameliorated", and 4.2\% (3/72, 95\% CI: 0.0-8.9) as "unchanged". Asked whether their lesion had healed meanwhile $45.8 \%(33 / 72$, 95\% CI: 34.0-57.6) of patients answered "no", "undecided", and "unknown", and 54.2\% (39/72, 95\% CI: 42.4-66.0) of patients answered "yes". Of patients, $61.1 \%(44 / 72$, 95\%CI: 49.6-72.6) argued that our conservative management policy is "valuable to test" or "understandable", 5.6\% (4/72, 95\% CI: 0.0-11.0) found it "incomprehensible" or "impracticable". Of our patients, $33.3 \%(24 / 72)$ did not answer.

Twenty-two (45.8\%) out of 48 patients who sent their questionnaire back reported at least one symptom: itching (18.8\%), a lump or pricking (both $10.4 \%$ ), pain $(8.3 \%)$, sore anus (8.3\%), bleeding (6.3\%), burning (baking), weeping, mucous (each 4.2\%). Symptoms appeared "once a month" (59.1\%, 13/22), “once a week” (27.3\%, 6/22), and "every day" (13.6\%, 3/22). Patients complied with our strict management policy "more than one week" $(37.5 \%$, 27/72, 95\% CI: 26.0-49.0), “one week” (25.0\%, 18/72, 95\% CI: 14.8-35.2), and "not at all” or "unknown" $(37.5 \%$, 95\% CI: 26.0-49.0).

Of our patients, 47.2\% (34/72, 95\% CI: 35.4-59.0) experienced no recurrences whereas 13.9\% (10/72, 95\%
Table 3 Patient characteristics at study entry

\begin{tabular}{ll}
\hline Number of patients & 72 \\
Males (\%) & $6 \mathrm{I}$ \\
Germans (\%) & 90 \\
Height (mean, cm) & 180 \\
Men & 166 \\
Women & \\
Weight (mean, kg) & 80 \\
Men & 65 \\
Women & \\
BMl & 24.9 \\
Men & 23.4 \\
Women & 43 \\
Age (mean, years) & 40 \\
Men & 46 \\
Women & \\
Profession (\%) & \\
Clerk & 42 \\
Self-employee & 26 \\
Trainee & 11 \\
Housewife & 8 \\
Pensioner & 7 \\
Worker & 6 \\
\hline
\end{tabular}

Abbreviation: $\mathrm{BMI}$, body mass index.

CI: 5.7-22.1) suspected a recurrence, 4.2\% (3/72) of patients did not know, and 34.7\% (25/72) of patients did not answer this question. Asked what kind of therapy followed their possible recurrence, patients answered: surgery (two patients), ointments and suppositories (two patients), and 13 patients remembered our anal cleaning policy which was adopted again. Comparing anal cleaning attitudes at start of study and six months later we found no change concerning use of dry toilet paper, wet wipes, shower, soap, or shower gel, besides a decline in use of wetted toilet paper ( $p=0.004$, exact significance two-tailed, McNemar test). On the contrary, use of shower and bath tube increased considerably ( $\mathrm{p}=0.01$, Wilcoxon signed-rank test).

\section{Discussion}

Our study is small in patient numbers compared to others $\mathrm{s}^{8,20,27}$ but has two advantages: data were gathered prospectively, and follow up period was short for all patients: six months (median, range: $2-13$ months) with no range extending up to $4,{ }^{8} 5,{ }^{20}$ or $12^{27}$ years. Disadvantages are: results were obtained by questionnaire only as with others ${ }^{20}$ due to the German insurance system which does not allow to see referred patients for follow-up, and regrettably only two thirds of 

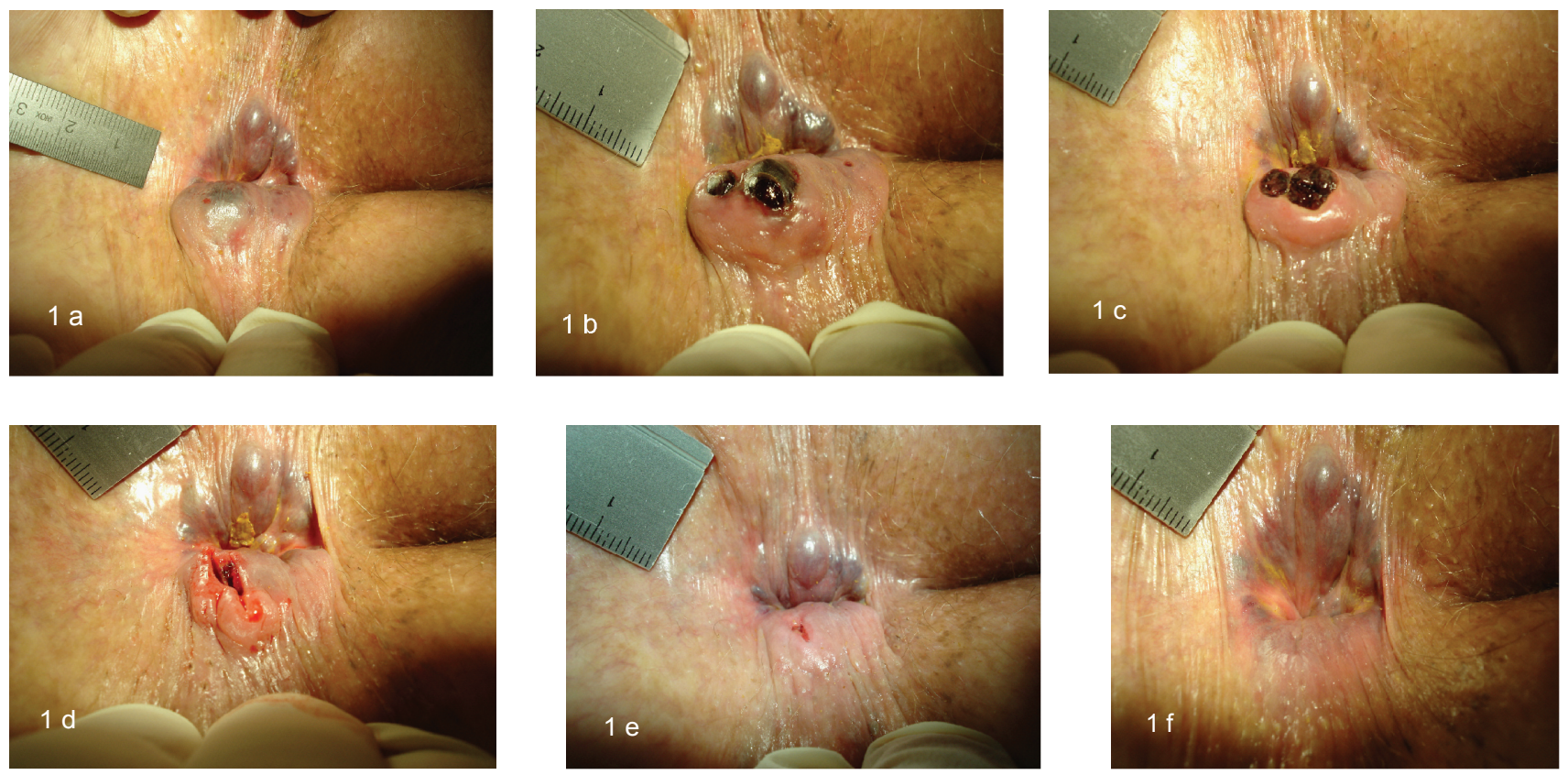

Figure I All six photos are taken from the same patient. In three-day intervals, they show the healing of a perforated and bleeding single thrombosed external hemorrhoid within nine days of a patient who consequently complied to our strict conservative management policy. A) Day 0: The patient is in knee-chest position, head left. Right-lateral of the anus parts of the uninflamed external hemorrhoidal plexus are protruding. Left-lateral there is edematous tissue with a dark spot (nonperforated thrombosed external hemorrhoid) with a subcutaneous clot. B) Day 3: Perforation and anal bleeding occurred in between. Right-lateral of the anus parts of an unaltered external hemorrhoidal plexus are seen. Left-lateral redness and edema of inflamed anal skin perforated by two black clots. C) Day 6: The right-lateral parts of the external hemorrhoidal plexus remain unchanged.The left-lateral clots are still at same position. D) Day 6: Both clots were taken out. A gaping lesion remains at former perforation site. E) Day 9: A 2-4 mm healing lesion is seen at former perforation site. At right-lateral, unchanged parts of the external hemorrhoidal plexus. F) Day 32: At follow-up four weeks later, the left-lateral perforation can hardly be seen. At right-lateral, the uninflamed subcutaneous external hemorrhoidal plexus appears unchanged.

patients $(48 / 72 ; 66.7 \%)$ sent their follow up questionnaire back. According to the intention-to-treat analysis we calculated these 24 nonresponders as drop-outs.

$\mathrm{TEH}$ is common in young persons ${ }^{8,20}$ which complies with our series mean age being 43 years. Incidence was higher in male than in female patients like with others 8,20 the ratio being $2: 1$. Similarly to other reports, ${ }^{17,27}$ half of our patients $(50.7 \%)$ had a prior history of TEH which might emphasize our suspicion that pruritus ani/perianal anitis ${ }^{1}$ is the determining precursor of $\mathrm{TEH}^{24,25}$ largely underdiagnosed according to type of proctological assessment. ${ }^{26}$ At follow-up, the most frequent complain in our series was itching (21.4\%). This signifies that anal inflammation ${ }^{1,24,25}$ is still a problem when TEH has healed.

Patients whose initial presentations were pain or bleeding with or without a lump were more like to be treated surgically ${ }^{27}$ if encountered within the first 72 hours. ${ }^{3,8,23}$ Since only $40.0 \%$ of our patients sought medical help within "some days" after onset of symptoms but the majority (60.0\%) later after "one week or more" most of them did not fulfill current prerequisites for surgery. ${ }^{3,8,16,20,23}$ Most of our patients sought medical help only when THE was healing. Early complications of surgery are postoperative bleeding, urinary retention, painful defecation, ${ }^{15,20}$ and abscess/fistula. ${ }^{20}$ Late complications include anal stenosis. ${ }^{16}$ Even though a possible advantage of surgery might be more rapid symptom resolution, lower incidence of recurrence, and longer remission intervals ${ }^{27}$ why should we expose our patients to such risks "since the condition is usually self-limiting and subsides in a few days to a week?"8

Recurrences were found to be less frequent after surgical $(6.3 \%)$ compared to conservative treatment $(25.4 \%)$ in a retrospective study. ${ }^{27}$ One cause might be that patients who had surgery timidly tried to avoid another operation and therefore neglected to present themselves to their surgeon again. The rate of suspected recurrence in our series was high (10/48, 21.3\%). Indeed typical complaints distinctive of recurrent TEH were rare: lump (10.4\%), pain (8.3\%), and bleeding $(6.3 \%)$. Therefore, recurrences with our patients seem rather unlikely but anal inflammation is supposable announced by itching (18.8\%), pricking (10.4\%), and a sore anus $(8.3 \%) .{ }^{24,25,28}$

Patient attitudes against our treatment policy did not influence therapeutic results since the majority of patients characterized it as "valuable to test" or "understandable" $(61.1 \%)$. These patients had no better results than those who 
found it "incomprehensible" or "impractical". After all, two young men had surgery. Both sought advice on the Internet about common treatments of TEH because they were discontent with our strict conservative management policy unlike our tolerating 73-year-old female patient (Figures 1A-F). Our study indicates that a strict conservative management policy for TEH can be successful since only 5.6\% of patients found it "incomprehensible" or "impractical". Randomized controlled trials with long follow ups are needed, which may ultimately result in current surgical management policies for TEH being abandoned.

\section{Disclosure}

The authors disclose no financial or nonfinancial competing interests as well as interpretation of data or presentation of information which may be influenced by personal relationships with other people or organizations. H.R. had the idea. All authors contributed to the design of the study and construction of the study protocol. O.G. was responsible for research. O.G. and H.R. saw the patients and asked them to complete their questionnaires before proctologic assessment and at follow-up. The findings were compiled into a PC study-documentation sheet after medical assessment of each patient. Results were discussed with all authors. Y.H. was responsible for statistical evaluations. O.G. wrote the first drafts which were revised by all authors. H.R. wrote the final draft.

\section{References}

1. Alexander-Williams J. Pruritus ani. Br Med J (Clin Res Ed). 1983; 287:159-160.

2. Hancock BD. ABC of colorectal diseases. Haemorrhoids. BMJ. 1992;304:1042-1044.

3. Janicke DM, Pundt MR. Surgical excision of symptomatic thrombosed external hemorrhoids is indicated within 48 to 72 hours of pain onset. Emerg Med Clin N Am. 1996;14:757-758.

4. Nelson LR. Treatment of anal fissure. BMJ. 2003;327:354-355.

5. Abramowitz L, Sobhani I, Benifla JL, et al. Anal fissure and thrombosed external hemorrhoids before and after delivery. Dis Colon Rectum. 2002;45:650-655.
6. Burkitt DP. Varicose veins, deep vein thrombosis, and haemorrhoids: epidemiology and suggested aetiology. Br Med J. 1972;2:556-561.

7. Gai F, Trecca A, Suppa M, et al. Hemorrhoidal thrombosis. A clinical and therapeutic study on 22 consecutive patients. Chir Ital. 2006;58:219-223.

8. Oh C. Acute thrombosed external hemorrhoids. Mt Sinai J Med. 1989;56:30-32.

9. Perrotti P, Antropoli C, Molino D, et al. Conservative treatment of acute thrombosed external hemorrhoids with topical nifedipine. Dis Colon Rectum. 2001;44:405-409.

10. Eisenstat T, Salvati EP, Rubin RJ. The outpatient management of acute hemorrhoidal disease. Dis Colon Rectum. 1979;22:315-317.

11. Arthur KE. Anal haematoma (coagulated venous succule or peri-anal thrombosis). Rev Med Panama. 1990;15:31-34.

12. Delaini GG, Bortolasi L, Falezza G, et al. Hemorrhoidal thrombosis and perianal hematoma: diagnosis and treatment. Ann Ital Chir. 1995; 66:783-785.

13. Iseli A. Office treatment of hemorrhoids and perianal hematoma. Aus Fam Physician. 1991;20:284-290.

14. Thomson H. The real nature of "perianal haematoma". Lancet. 1982; 8296:467-468.

15. Nieves PM, Perez J, Suarez JA. Hemorrhoidectomy - how I do it: experience with the St.Mark's Hospital technique for emergency hemorrhoidectomy. Dis Colon Rectum. 1977;20:197-201.

16. Barrios G, Khubchandani M. Urgent hemorrhoidectomy for hemorrhoidal thrombosis. Dis Colon Rectum. 1979;22:159-161.

17. Brearly S, Brearly R. Perianal thrombosis. Dis Colon Rectum. 1988;31:403-404.

18. Nagle D, Rolandelli RH. Primary care office management of perianal and anal diseases. Primary Care. 1996;23:609-620.

19. Metcalf A. Anorectal disorders. Postgrad Med. 1995;89:81-94.

20. Jongen J, Bach S, Stuebinger SH, et al. Excision of thrombosed external hemorrhoid after local anesthesia. A retrospective evaluation of 340 patients. Dis Colon Rectum. 2003;46:1226-1231.

21. Sakulsky SB, Blumenthal JA, Lynch RH. Treatment of thrombosed hemorrhoids by excision. Am J Surg. 1970;120:537-538.

22. Grosz CR. A surgical treatment of thrombosed external hemorrhoids. Dis Colon Rectum. 1990;33:249-250.

23. Zuber TJ. Hemorrhoidectomy for thrombosed external hemorrhoids. Am Fam Physician. 2002;65:1629-1632.

24. Rohde H. Routine anal cleansing, so-called hemorrhoids, and perianal dermatitis: cause and effect? Dis Colon Rectum. 2000;43:561-562.

25. Rohde H. Schädigung der Analhaut durch Nassreinigung. Dtsch Med Wschr. 2005;130:974.

26. Rohde H. Diagnostic errors. Lancet. 2000;356:1278.

27. Greenspon J, Williams St B, Young HA, et al. Thrombosed external hemorrhoids: outcome after conservative or surgical management. Dis Colon Rectum. 2004;47:1493-1498.

28. Kuehn H G, Gebbensleben O, Hilger Y, Rohde H. Relationship between anal symptoms and anal findings. Int J Med Sci. 2009;6:77-84.
Clinical and Experimental Gastroenterology

\section{Publish your work in this journal}

Clinical and Experimental Gastroenterology is an international, peerreviewed, open access journal, publishing all aspects of gastroenterology in the clinic and laboratory, including: Pathology, pathophysiology of gastrointestinal disease; Investigation and treatment of gastointestinal disease; Pharmacology of drugs used in the alimentary tract;

\section{Dovepress}

Immunology/genetics/genomics related to gastrointestinal disease. This journal is indexed on CAS. The manuscript management system is completely online and includes a very quick and fair peer-review system. Visit http://www.dovepress.com/testimonials.php to read real quotes from published authors. 